\title{
The response of phyllodes tumor of the breast to anticancer therapy: An in vitro and ex vivo study
}

\author{
ALICJA URBANIAK $^{1^{*}}$, FARIBA JOUSHEGHANY ${ }^{2 *}$, YOUZHONG YUAN ${ }^{2}$, SERGIO PIÑA-OVIEDO $^{2}$, \\ ADAM HUCZYŃSKI ${ }^{3}$, MAGDALENA DELGADO ${ }^{1}$, THOMAS KIEBER-EMMONS $^{2}$, \\ BEHJATOLAH MONZAVI-KARBASSI ${ }^{2}$, and TIMOTHY C. CHAMBERS ${ }^{1}$
}

\begin{abstract}
Departments of ${ }^{1}$ Biochemistry and Molecular Biology, and ${ }^{2}$ Pathology, University of Arkansas for Medical Sciences, Little Rock, AR 72205, USA; ${ }^{3}$ Department of Bioorganic Chemistry, Faculty of Chemistry, Adam Mickiewicz University, Umultowska 89b, 61-614 Poznań, Poland
\end{abstract}

Received March 8, 2019; Accepted July 12, 2019

DOI: $10.3892 / \mathrm{ol} .2019 .10823$

\begin{abstract}
Phyllodes tumors of the breast (PTB) are uncommon stromal-epithelial neoplasms, with the main recommended treatment being surgical removal. However, even with adequate resection, the risk of recurrence in the malignant form remains as high as $40 \%$, and there is no recognized consensus on the most effective drugs for PTB. In the present study, an ex vivo model of malignant phyllodes and derived primary cell cultures were used to evaluate the effectiveness of a panel of different drugs, including the $\mathrm{Bcl}-2 / \mathrm{Bcl}-\mathrm{xL}$ inhibitor ABT-263, salinomycin (SAL), doxorubicin (DOX), paclitaxel (TAX), vincristine (VCR), colchicine (COL) and cisplatin (CIS). ABT-263, SAL and DOX were highly effective towards phyllodes spindle cells when assessed in the ex vivo model, contributing to $~ 98 \%$ tumor cell death. Furthermore, ABT-263 was highly selective for tumor cells in this system, and exhibited little toxic effect on adjacent normal epithelial cells. Furthermore, consistent with findings in the ex vivo model, ABT-263 was significantly less toxic towards MCF 10A non-tumorigenic breast epithelial cells compared with SAL and DOX. A conditional reprogramming strategy was subsequently used, involving Rho kinase inhibition, to successfully generate primary phyllodes tumor cells that could be cultured
\end{abstract}

Correspondence to: Professor Timothy C. Chambers, Department of Biochemistry and Molecular Biology, University of Arkansas for Medical Sciences, 4301 W. Markham, Little Rock, AR 72205, USA

E-mail: chamberstimothyc@uams.edu

Professor Behjatolah Monzavi-Karbassi, Department of Pathology, University of Arkansas for Medical Sciences, 4301 W. Markham, Little Rock, AR 72205, USA

E-mail: KarbassiBehjatolahM@uams.edu

${ }^{*}$ Contributed equally

Key words: malignant phyllodes tumor, breast, pathology, chemotherapy, ex vivo for several passages. The primary cells were sensitive to DOX with an $\mathrm{IC}_{50}$ of $0.40 \pm 0.07 \mu \mathrm{M}$ in a standard viability assay and the preliminary results were obtained indicating sensitivity to ABT-263 and SAL. The present study demonstrated the feasibility of using explants and primary cells for drug discovery, selectively targeting PTB cells.

\section{Introduction}

Phyllodes tumor (PT) was first described in 1838 by the German physician Johannes Müller as cystosarcoma phyllodes (1). The name 'phyllodes' derives from the Latin Phyllodium and means 'leaf-like' which relates to its morphology under the microscope (2). Phyllodes tumors of the breast (PTB) are rare, accounting for less than $1 \%$ of breast neoplasms overall (3) which translates into about 500 cases diagnosed in the USA annually (4). The mean age of diagnosed patients is 40 years old, which is lower than patients with conventional breast carcinomas (2). PTBs are classified into three categories: Benign, borderline and malignant (5). Malignant lesions with the ability to metastasize distantly can be observed in $30 \%$ of cases (6). Recommended treatment includes wide excision with clear margins and prognosis depends on the histological analysis of the mesenchymal component (7). Benign histology, negative tumor margins and an absence of residual disease after initial treatment and radiation therapy have been determined as favorable prognostic factors (8). The main problem in the treatment and management of PTB is its local recurrence in both benign and malignant cases (2). With no prospective trials of chemotherapy for malignant phyllodes tumors (MPT), the utility of drug treatment remains unclear and requires more detailed case-by-case studies $(2,9)$. Although routine chemotherapy is not a standard treatment for MPT it can be considered for large tumors or when secondary structures such as the chest wall are involved (2). So far neoadjuvant doxorubicin (DOX) with dacarbazine versus no medical therapy has been studied in a small observational biased trial where no positive outcome on relapse-free survival has been observed (10). The other approach was described by Hashimoto el al (11) where pre-operative chemoembolization of a large MPT of the breast (MPTB) led to successful avoidance of skin grafting after excision. 
The development of antitumor drugs has depended largely on cancer cell lines and animal models, and these have played an important role in rapid screening and identification of candidates for further evaluation. The NCI 60 cell line panel, for example, is a popular screening tool (12). Cell lines offer several advantages including ease of handling and convenient restoration from frozen storage, and their ability to serve as an essentially unlimited source of large quantities of relatively homogenous cells (13). However, they also have drawbacks that limit their usefulness. For example, cell culture models lack the tumor microenvironment which is known to critically impact therapeutic response; the engraftment of tumor cells in xenograft models relies on the host response in a non-native setting; and transgenic mouse models do not always reproduce aspects of human disease $(14,15)$. In addition, in the case of a heterogeneous disease such as breast cancer, single models do not represent the various subtypes observed clinically. Furthermore, cell lines are prone to undergo phenotypic and genetic drift, such that ostensibly identical cell lines may differ substantially from one laboratory to another (13). Many of these problems can be circumvented through the use of explant cultures, where pieces of fresh tumor are incubated ex vivo on media-soaked sponges. Importantly, explants retain tissue architecture, degrees of cellularity, and specific tumor markers found in the originating tumor (16-18). Such a system has tremendous general promise for breast cancer drug discovery and biomarker development, with the potential to accelerate personalized treatmentoptions. However, adrawback of explants is their limited timespan of utility of 3-4 days from excision.

The generation of primary cell lines from epithelial-derived tumors has historically been difficult because the cells can only be cultured for a few passages before ceasing proliferation and undergoing senescence (19). Although primary cells can be immortalized through the introduction of oncogenes or telomerase (20), such cells do not retain lineage commitment, and they exhibit aberrant retinoblastoma and $\mathrm{p} 53$ pathways and display abnormal growth or differentiation potential. Recently it has been shown that human epithelial cells, from a variety of sources both normal and tumor, can bypass senescence and be cultured long-term if grown on irradiated fibroblast feeder cells in the presence of the Rho kinase inhibitor Y-27632 (21-23). These 'conditionally reprogrammed' cells (CRCs) represent a powerful and novel model for breast cancer drug discovery, and serve as a more durable complement to explant cultures.

In the present study, we evaluated the effectiveness of seven drugs namely the Bcl-2/Bcl-xL inhibitor ABT-263, salinomycin (SAL), DOX, paclitaxel (TAX), vincristine (VCR), colchicine (COL), and cisplatin (CIS) in an ex vivo model of PTB (24-26). These drugs were selected because they represent a cross section of standard drugs used for breast cancer treatment (DOX, TAX, CIS) (24) or are related in mechanism (VCR and COL, which are microtubule inhibitors like TAX) (25). In addition, we tested ABT-263 since it is being advanced clinically as a direct inducer of intrinsic apoptosis (26), and we also examined SAL since we have shown previously that it is effective against ductal breast cancer in an ex vivo model (27). We also successfully generated primary PTB cells from the same tumor specimen and have initiated drug testing in this system. The results presented demonstrate the feasibility of using explants and primary cells for drug discovery selectively targeting PTB cells.

\section{Materials and methods}

Materials. TAX (cat. no. T1912), COL (cat. no. C-9754), DOX (cat. no. PHR1789) and CIS (cat. no. 479306) were purchased from Sigma-Aldrich (Darmstadt, Germany). VCR sulfate (cat. no. SC-201434) was obtained from Santa Cruz Biotechnology (Dallas, TX, USA) and ABT-263 (Navitoclax) (cat. no. A3007) from ApexBio (Houston, TX, USA). SAL sodium salt was obtained from commercially available veterinary premix SACOX $^{\circledast}$ following acidic extraction using the procedure described previously (28).

Preparation and culture of tissue slices. Breast tumor tissue was provided by the Cooperative Human Tissue Network (CHTN, https://www.chtn.org/), a National Cancer Institute supported resource. Other investigators may have received samples from these same tissue specimens. Fresh breast cancer tissue was collected from a 33 year old African American female patient diagnosed with $16.3 \mathrm{~cm}$ malignant phyllodes tumor of the breast. Immediately after surgical resection, a portion of the specimen was transported and stored in fresh RPMI 1640 medium on ice for $24 \mathrm{~h}$ prior to use. The day after surgical removal, slices were prepared from the specimen according to our previously established methodology (27) based on the studies of Van der Kuip et al (29). Briefly, $200 \mu \mathrm{m}$ slices were cut in sterile, cold PBS (cat. no. 21-030-CM, Corning, Manassas, VA, USA) supplemented with $1 \%$ antibiotic/antimycotic solution (cat. no. A5955, Sigma-Aldrich, Deisenhofen, Germany) using a microtome with vibrating blade (Leica Biosystems VT1200, Nussloch, Germany). Blades were steam sterilized before use. Several individual slices from different parts of the specimen were immediately placed into embedding cassettes (cat. no. 27158-2B and cat. no. 27154-1, Ted Pella, Redding, CA, USA) and fixed in $10 \%$ neutral-buffered formalin for $24 \mathrm{~h}$ and further stored in $70 \%$ ethanol prior to processing. The remaining slices were distributed in separate wells of a 24 well plate in $1 \mathrm{ml}$ of Mammary Epithelial Cell Basal Medium (cat. no. C-21215, Promo Cell, Heidelberg, Germany) supplemented with Mammary Epithelial Cell Supplement Pack (cat. no. C-39110, Promo Cell, Heidelberg, Germany), $100 \mu \mathrm{g} / \mathrm{ml}$ gentamicin (cat. no. G1397, Sigma-Aldrich, Deisenhofen, Germany) and $0.05 \mu \mathrm{g} / \mathrm{ml}$ amphotericin B (cat. no. A2942, Sigma-Aldrich, Deisenhofen, Germany). The plate was incubated at $37^{\circ} \mathrm{C}$ in a constant atmosphere of $5 \% \mathrm{CO}_{2}$ on a shaking platform at 150 rpm (Orbi-Shaker Jr, Benchmark Scientific, Sayreville NJ, USA). After $24 \mathrm{~h}$ to allow tissue equilibration, treatment with $0.2 \%$ DMSO or ABT-263, SAL, DOX, TAX, VCR, COL and CIS, at concentrations of 2,8 and $16 \mu \mathrm{M}$ was initiated for $72 \mathrm{~h}$. Concentrations were selected based on previous findings of van der Kuip et al (29). The medium was changed every $24 \mathrm{~h}$. Tissue slices were then fixed and stored as described above.

Immunohistochemical staining. Immunohistochemical staining of untreated, vehicle- and drug-treated samples was performed by UAMS Experimental Pathology Core. For histopathological examination, paraffin embedded sections $(4 \mu \mathrm{m})$ were stained with hematoxylin and eosin (H\&E) (cat. no. 7231, Richard-Allan Scientific, Thermo Fisher Scientific, Waltham, 
MA, USA). Dako DAB+ (cat. no. K3468, Dako Liquid, Carpenteria, CA, USA) was used for 3 min prior to counterstaining with hematoxylin. Immunohistochemical staining for Ki-67 (1:100, rabbit monoclonal anti-Ki67, cat. no. ab16667, Abcam, Cambridge, MA, USA) was performed with biotinylated goat anti-rabbit second antibody (1:400) (cat. no. BA-1000, Vector, Burlingame, CA, USA) followed by detection using the Vectastain ABC Elite detection system for 30 min (cat. no. PK-6100, Vector, Burlingame, CA, USA). Epitope retrieval (cat. no. S1699, Target Retrival Solution, Dako, Carpenteria, CA, USA) was achieved prior to the staining in a decloaking chamber for 20 min (Biocare medical). Dead or dying tumor cells were quantified manually based on changes in cell and nuclear morphology. Quantification was performed employing a Nikon Eclipse E600 microscope at 4x, 10x and 20x. Specifically, tumor cells which were markedly reduced in size and/or showed nuclear fragmentation were scored. Effects of drugs on normal ductal epithelial cells were similarly assessed by evaluating cellular and nuclear morphology of individual cells as well as the extent to which epithelial organization was disrupted. A total of at least 50 cells were examined per condition each of which was conducted in triplicate.

Generation of primary phyllodes cells. To establish primary PTB cells, a procedure for conditional reprogramming of cells (CRC) was followed (21). Briefly, the remaining tumor specimen was cut into 1-mm thick slices which were dissociated by incubating in a mixture of $0.25 \mathrm{x}$ Collagenase/Hyaluronidase and 0.25x Dispase (cat. no. 7919, cat. no. 7913, Stem Cell Technologies, Vancouver, Canada) diluted in F-medium 3:1 (v/v) F-12 Nutrient Mixture (Ham)/Dulbecco's modified Eagle's medium (cat. no. 11765-054, Gibco, Grand Island, NY, USA), 5\% fetal bovine serum (cat. no. FP-0500-A, Atlas Biologicals, Fort Collins, CO, USA), $24 \mu \mathrm{g} / \mathrm{ml}$ adenine (cat. no. A2786-5G, Sigma-Aldrich, Deisenhofen, Germany), $8.4 \mathrm{ng} / \mathrm{ml}$ cholera toxin (cat. no. C8052-.5MG, Sigma-Aldrich, Deisenhofen, Germany), $10 \mathrm{ng} / \mathrm{ml}$ epidermal growth factor (cat. no. PHG0311, Life Technologies, Waltham, MA, USA), 1xantibiotic/antimycoticsolution(cat.no.A5955,Sigma-Aldrich, Deisenhofen, Germany), $0.4 \mu \mathrm{g} / \mathrm{ml}$ hydrocortisone (cat. no. H4001, Sigma-Aldrich, Deisenhofen, Germany), $5 \mu \mathrm{g} / \mathrm{ml}$ insulin (cat. no. 128-100, Cell Applications, Inc., San Diego, CA, USA) for $2 \mathrm{~h}$. Mouse fibroblast conditioned medium was prepared from Swiss 3T3-J2 mouse fibroblasts (cat. no. EF3003, Kerafast, Inc., Boston MA, USA) cultured in DMEM supplemented with $10 \%$ bovine calf serum (iron supplemented without gamma-irradiation or heat-inactivation from GE Healthcare, Little Chalfont, England) following manufacturer instructions. Dissociated cells were then seeded in 12-well tissue culture plates in a mixture of F-medium and mouse fibroblast culture supernatant at a ratio of 4:1 (v/v) supplemented with $5 \mu \mathrm{M}$ Rho kinase inhibitor (cat. no. ALX-270-333-M005, Enzo Life Sciences, Farmingdale, NY, USA). Cells were maintained at $37^{\circ} \mathrm{C}$ in a humidified incubator with $5 \% \mathrm{CO}_{2}$. Colonies became visible after 5 to 7 days and cells were passaged after two weeks (approximately $10^{5}$ cells) and expanded after reaching 80 to $90 \%$ confluence. An image of cells at passage 6 was recorded using phase contrast microscopy using an EVOS FL Auto Cell Imaging System (Thermo Fisher Scientific).
MCF-7, MDA-MB-231 and MCF 10A cell lines. Human MCF-7 mammary gland adenocarcinoma cells originally isolated from a 69 year old Caucasian woman with several characteristics of differentiated mammary epithelium were cultured in Eagle's Minimum Essential Medium (EMEM) (cat. no. 30-2003, ATCC, Manassas, VA, USA) supplemented with $10 \%(\mathrm{v} / \mathrm{v})$ heat-inactivated fetal bovine serum (FBS) (cat. no. FP-0500-A, Atlas Biologicals, Fort Collins, CO, USA), and 1\% Penicillin/Streptomycin Solution 100x (cat. no. 30-002-Cl, Corning, Manassas, VA, USA). Human MDA-MB-231 mammary gland adenocarcinoma cells isolated as one of a series of breast tumor lines from pleural effusions of a 47 year old Caucasian female were cultured in DMEM/Ham's Nutrient Mixture F12 1:1 (cat. no. 51445C, Sigma-Aldrich, Deisenhofen, Germany) supplemented with $5 \%(\mathrm{v} / \mathrm{v})$ heat-inactivated fetal bovine serum (FBS) (cat. no. FP-0500-A, Atlas Biologicals, Fort Collins, CO, USA), $1 \%$ Penicillin/Streptomycin Solution 100x (cat. no. 30-002-Cl, Corning, Manassas, VA, USA) and $1 \mathrm{mM}$ L-Glutamine (cat. no. 25005-Cl, Corning, Manassas, VA, USA). Both cell lines were tested via short tandem repeat profiling in July 2018 by Genetica DNA Laboratories (Burlington, NC, USA) and verified as authentic, giving a 100\% match when compared to the known reference profile (30). Human MCF $10 \mathrm{~A}$ mammary epithelial cells originally isolated from 36 year old Caucasian women were purchased from ATCC, Manassas, VA, USA (cat. no. CRL-10317) and cultured in Mammary Epithelial Cell Basal Medium (cat. no. C-21215, Promo Cell, Heidelberg, Germany) supplemented with Mammary Epithelial Cell Supplement Pack (cat. no. C-39110, Promo Cell, Heidelberg, Germany), $100 \mu \mathrm{g} / \mathrm{ml}$ gentamicin (cat. no. G1397, Sigma-Aldrich, Deisenhofen, Germany) and $0.05 \mu \mathrm{g} / \mathrm{ml}$ amphotericin B (cat. no. A2942, Sigma-Aldrich, Deisenhofen, Germany).

Flow cytometry analysis. EpCAM expression was assessed by binding of FITC-conjugated anti-EpCAM monoclonal mouse anti-human antibody (1:10 dilution) (cat. no. 324203, Biolegend, San Diego, CA, USA). MCF-7, MDA-MB-231 and human primary phyllodes cells (about $5 \times 10^{4}$ cells) were harvested with GIBCO $^{\circledR}$ enzyme-free cell-dissociation buffer (cat. no. 13151-014, Life Technologies, Grand Island, NY, USA), washed with flow-cytometry buffer (PBS containing 1\% BSA) and incubated with FITC-conjugated anti-EpCAM antibody (1:10 dilution) in the same buffer for 30 min followed by washing twice with flow-cytometry buffer. Cells were fixed with $1 \%$ (final concentration) PFA. Acquisition and analysis of data was performed by UAMS Flow Cytometry Core using a LSRFORTESSA flow cytometer (BD Biosciences, San Jose, CA, USA) and FlowJo ${ }^{\circledR}$ software (FlowJo LLC, Ashland, OR, USA).

Cell viability assay. A 3-(4,5-dimethylthiazol-2-yl)2,5-dimethylthiazol-2-yl)-2,5-diphenyltetrazolium bromide (MTT)-based assay $(31,32)$, was used to evaluate the effect of drugs on the viability of primary phyllodes cells and MCF $10 \mathrm{~A}$ cells. Phyllodes cells $\left(0.35 \times 10^{4} /\right.$ well $)$ in $100 \mu \mathrm{l}$ of F-medium were seeded in 96-well plates (TPP, Trasadingen, Switzerland). After $24 \mathrm{~h}$ cells were treated with DOX in the following concentrations: $1,3,10,30,100,300,1,000,3,000$ and $10,000 \mathrm{nM}$, as well as SAL and ABT-263 at a single concentration of $10 \mu \mathrm{M}$ 

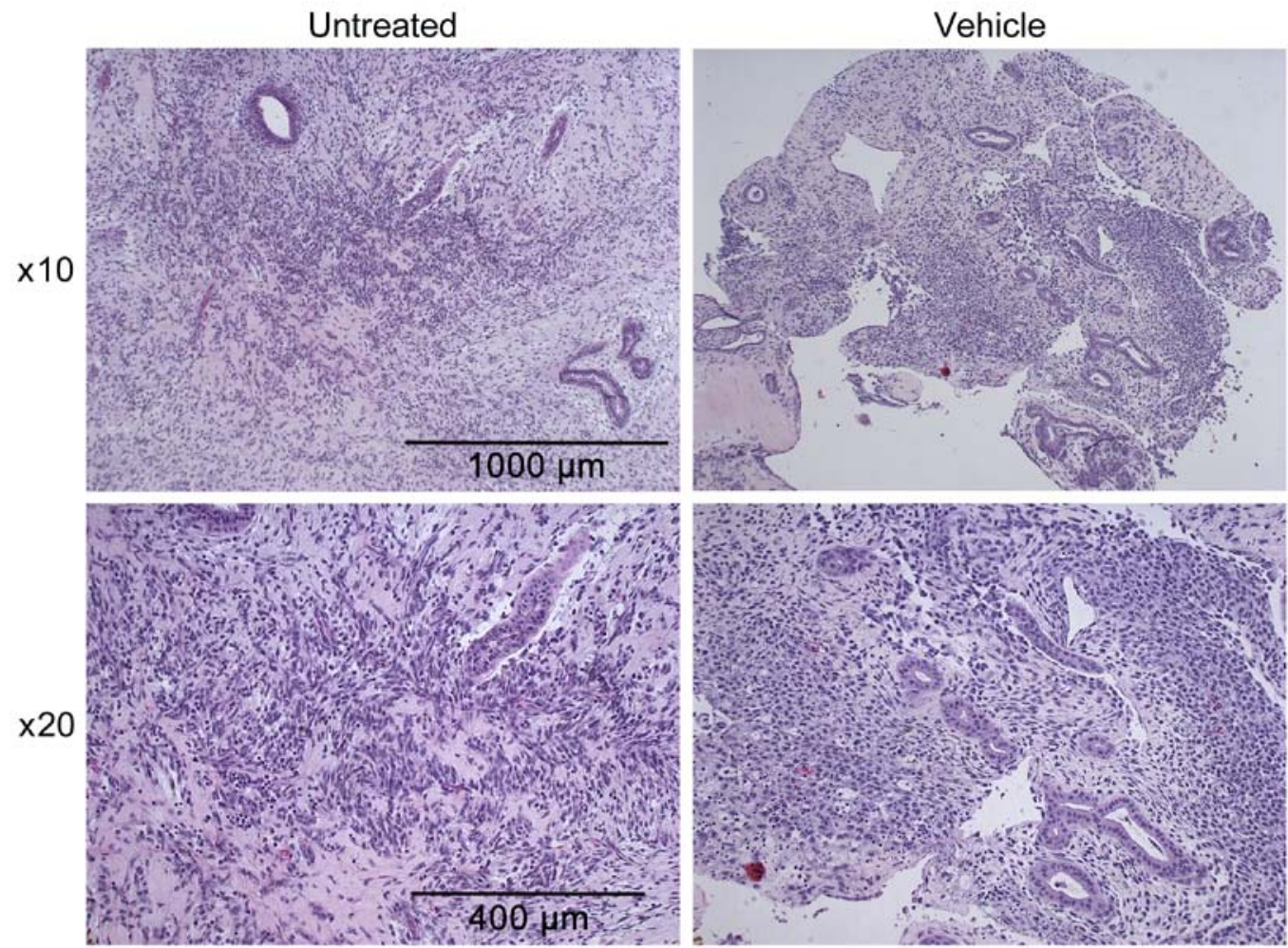

Figure 1. Morphology of PTB. Representative haematoxylin and eosin stained sections of breast phyllodes tumor fixed $24 \mathrm{~h}$ after surgery (untreated) and after $72 \mathrm{~h}$ of culturing in medium in the presence of $0.2 \%$ DMSO (vehicle). Two magnifications, 10x and 20x, are presented. PTB, phyllodes tumors of the breast.

for $96 \mathrm{~h}$ with control cells receiving vehicle (0.1\% DMSO) alone. The experiment was performed in triplicate. MCF 10 A cells $\left(1 \times 10^{4} /\right.$ well $)$ in $100 \mu$ l of MEGM were seeded in 96-well plates (TPP, Trasadingen, Switzerland) and after $24 \mathrm{~h}$ treated with DOX, SAL and ABT-263 at the following concentrations: $1,3,10,30,100,300,1,000,3,000$ and $10,000 \mathrm{nM}$ (of DOX and SAL) and 300, 1,000, 1,200, 1,400, 1,600, 1,800, 2,200, $3,000 \mathrm{nM}$ of ABT-263 for $96 \mathrm{~h}$ with control cells receiving vehicle $(0.1 \%$ DMSO) alone. The experiment was performed in quadruplicate. After treatment, $10 \mu \mathrm{l}$ of MTT solution $(5 \mathrm{mg} / \mathrm{ml}$, cat. no. M2128, Sigma-Aldrich, Deisenhofen, Germany) was added to each well, and the plate was incubated at $37^{\circ} \mathrm{C}$ for $4 \mathrm{~h}$ in a humidified $5 \% \mathrm{CO}_{2}$ incubator. Medium was then aspirated and $150 \mu \mathrm{l}$ of DMSO was added to each well and the plate agitated on a shaking platform $(150 \mathrm{rpm})$ for $10 \mathrm{~min}$. Absorbance was recorded at $540 \mathrm{~nm}$ using a BioTek Plate Reader. Inhibition of formation of colored MTT formazan was taken as an index of cytotoxicity activity. $\mathrm{IC}_{50}$ values were determined by non-linear regression analysis using GraphPad Prism 6 for Windows (GraphPad Software).

Statistical analysis. 1-way ANOVA with Tukey's multiple comparisons test was used to determine statistically significant differences between means in experimental groups. Data are presented as a mean $\pm \mathrm{SD}$. For ex vivo experiments three biological replicates were performed for every drug concentration. Cell viability assay employing primary phyllodes cells was performed in triplicate, whereas MCF 10 A cells in quadruplicate. EpCAM staining was performed in duplicate. Statistical analysis was performed using GraphPad Prism 6 for Windows (GraphPad Software, Inc., San Diego, CA, USA).

\section{Results}

Screening of cancer chemotherapeutics in an ex vivo model of MPTB. A single specimen of fresh, unfrozen MPTB was obtained, sliced, incubated with select drugs, and subjected to H\&E staining, as described in Materials and methods. A representative image of an untreated MPTB section fixed and stained immediately upon receipt is shown in Fig. 1 (left panel). The presence of a biphasic neoplasm made of neoplastic epithelial and mesenchymal (spindle cell) elements is consistent with PT. This was confirmed by the CHTN pathology report which subsequently became available. The report indicated that the tumor showed biphasic fibroepithelial growth with pushy lobulated margins, hypercellular stroma with leaf-like architecture, stromal outgrowth, moderate to severe nuclear atypia, high mitotic rate, and focal necrosis. Importantly, the overall morphological features and tumor cell viability remained unchanged in vehicle treated samples at $72 \mathrm{~h}$ (Fig. 1, right panel) compared to the untreated original tumor sample (Fig. 1, left panel), indicating that incubation had no deleterious effects. Next, explant cultures were treated with drugs including ABT-263, SAL, DOX, TAX, VCR, COL, and CIS. Representative images of H\&E stained slices at 20x magnification are presented in Fig. 2 for the most active compounds ABT-263, SAL, DOX, and in Fig. S1 for the other compounds. Healthy versus dead or drug-affected tumor or normal epithelial cells were distinguished and quantified. As evident from representative images in Fig. 2 and the corresponding quantitation in Fig. 3, ABT-263, SAL and DOX treatment caused marked tumor cell death in a dose-dependent manner compared to vehicle treated slices (Fig. 3). Of additional interest, treatment with ABT-263 had 

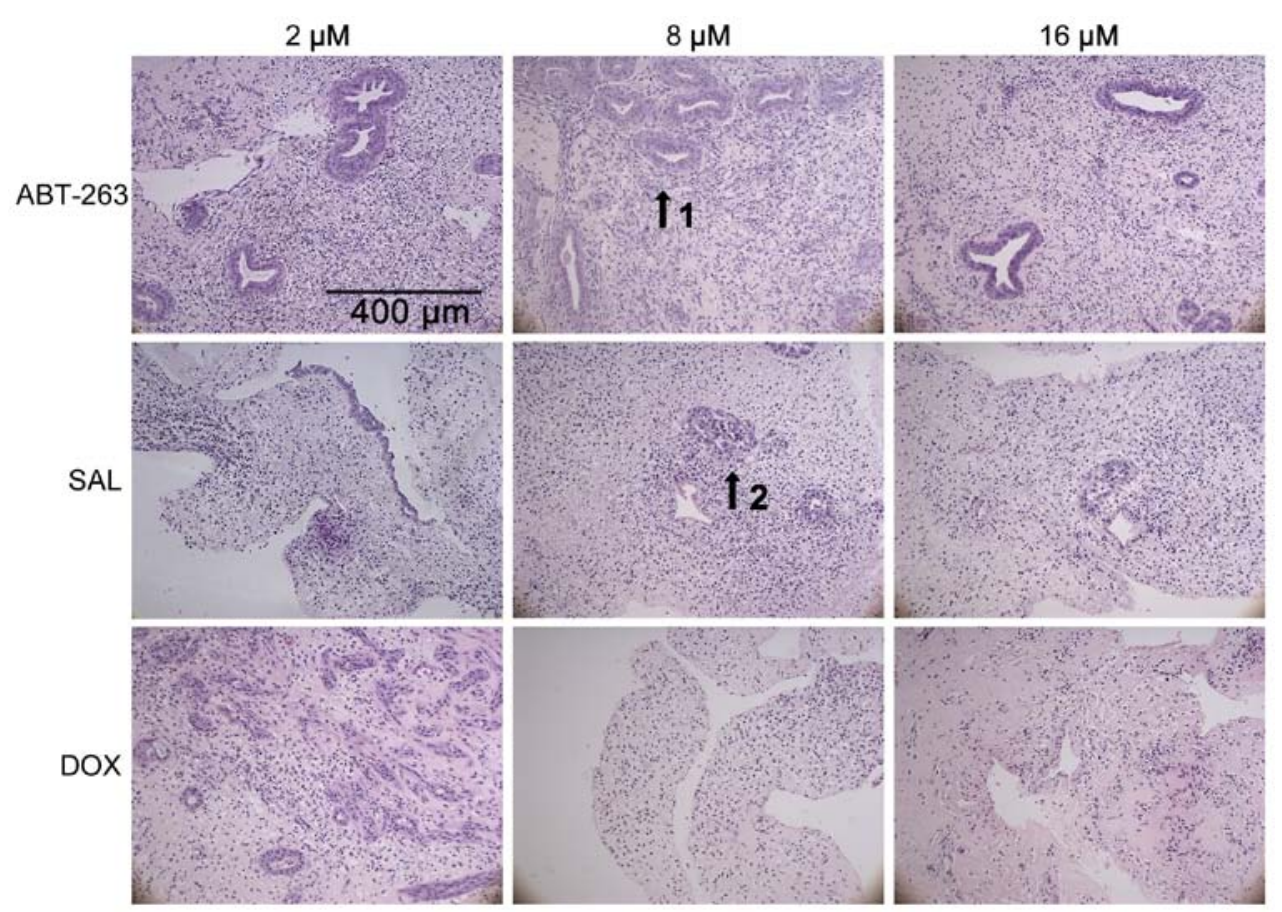

Figure 2. Cytotoxic effects of ABT-263, SAL and DOX on breast phyllodes tumor in an ex vivo model of PTB. Haematoxylin and eosin stain of representative sections at 20x magnification. Arrows indicate epithelial ducts. SAL, salinomycin; DOX, doxorubicin; PTB, Phyllodes tumors of the breast.

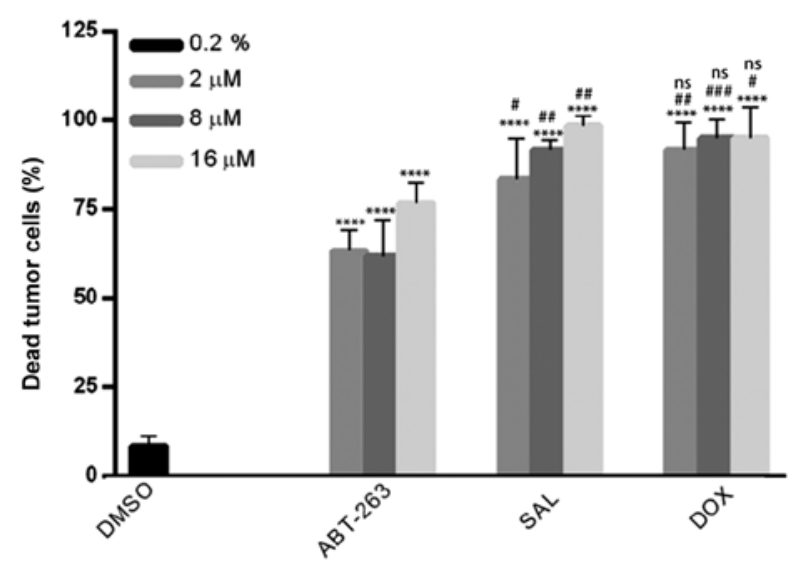

Figure 3. Quantification of dead tumor cells. Dead PTB cells were quantified in haematoxylin and eosin stained tumor sections. Statistical analysis using one-way ANOVA of effects on death of PTB: DMSO versus drugs ${ }^{* * * * * *} \mathrm{P} \leq 0.0001 ; \mathrm{ABT}-263$ versus $\mathrm{SAL}{ }^{\#} \mathrm{P} \leq 0.05,{ }^{\# \#} \mathrm{P} \leq 0.01 ; \mathrm{ABT}-263$ versus DOX ${ }^{\# P} \mathrm{P} \leq 0.05,{ }^{\# \#} \mathrm{P} \leq 0.01,{ }^{\# \#} \mathrm{P} \leq 0.001 ; \mathrm{SAL}$ versus DOX ns-no statistically significant differences have been observed. PTB, Phyllodes tumors of the breast; SAL, salinomycin; DOX, doxorubicin.

very little to no toxic effect on normal epithelial cells regardless of the dose (Fig. 2). This is evident from examination of the images in Fig. 2, where ducts retained their normal architecture and cellularity even with the highest tested ABT-263 concentration (indicated by arrow 1), and from quantitative assessment of toxicity (Table I). In contrast, the anti-tumor activities of SAL and DOX were accompanied by significant toxic effects on normal epithelial cells, particularly after DOX treatment (arrow 2 in Fig. 2; Table I).

Treatment of PTB explant cultures with TAX, VCR, COL, and CIS was also performed under similar conditions (Fig. S1A). However, none of these drugs produced significant effects on the tumor cells thus quantification and statistical analysis of dead tumor cells was not performed.

Additionally, sections from paraffin embedded tissue were analyzed by immunohistochemical staining for $\mathrm{Ki}-67$, which is an established marker of cell proliferation (33). Representative images at 20x magnification are presented in Fig. 4 and Fig. S1B. In untreated tumor sections, the Ki-67 proliferation index was very low $(<5 \%)$, in a good agreement with a previous study (34), whereas strong Ki-67 immunolabeling was observed in some of the ductal epithelial cells (Fig. S1B). Ductal epithelial Ki-67 immunolabeling was largely maintained after ABT-263 treatment but was not observed after treatment with SAL or DOX (Fig. 4), consistent with the differential effects of the drugs on normal cells.

In order to further assess the selectivity of ABT-263 towards tumor versus normal cells compared to SAL and DOX, both of which appeared less discriminate in the ex vivo model, we performed MTT viability assay on the MCF 10A cell line. These are non-tumorigenic mammary epithelial cells commonly used as a surrogate for normal mammary cells. As shown in Fig. 5, $\mathrm{IC}_{50}$ values were ABT-263, $1.60 \pm 0.03 \mu \mathrm{M}$; SAL, $0.11 \pm 0.03 \mu \mathrm{M}$; and DOX, $0.02 \pm 0.01 \mu \mathrm{M}$. Thus MCF $10 \mathrm{~A}$ cells are much more sensitive to SAL (20x) and DOX (120x) compared to ABT-263, in good agreement with observations made in the ex vivo model (Fig. 2 and Table I).

Generation of primary phyllodes cells. To further investigate the response of PTB cells to anticancer drugs we sought to generate primary cells from the MPTB we obtained. This was facilitated by recent advances in the preparation of primary cells through Rho kinase inhibition and the use of fibroblast feeder cells or conditioned medium derived therefrom $(21,22)$. An image from a phase contrast microscope of the cells derived from the MPTB tissue using this procedure is shown in Fig. 6 . 
Table I. Toxic effect of 2,8 and $16 \mu \mathrm{M}$ of ABT-263, SAL and DOX on normal epithelial cells in the ex vivo model (n=3).

\begin{tabular}{|c|c|c|c|c|c|c|c|c|c|}
\hline \multirow[b]{4}{*}{ Drug } & \multicolumn{9}{|c|}{ Concentration $[\mu \mathrm{M}]$} \\
\hline & \multicolumn{3}{|c|}{2} & \multicolumn{3}{|c|}{8} & \multicolumn{3}{|c|}{16} \\
\hline & \multicolumn{9}{|c|}{ Sample number } \\
\hline & 1 & 2 & 3 & 1 & 2 & 3 & 1 & 2 & 3 \\
\hline ABT-263 & 0 & 0 & 0 & 0 & 0 & $\dagger$ & 0 & 0 & 1 \\
\hline SAL & 2 & 2 & 3 & 3 & 2 & $\dagger$ & 3 & 3 & 3 \\
\hline DOX & 3 & 3 & 1 & 3 & 3 & 3 & 3 & 3 & 3 \\
\hline
\end{tabular}

The toxic effect was estimated in H\&E stained slides according to the following scale: 0 -no effect/intact ( $0 \%$ affected cells); 1 -mild ( $<25 \%$ affected cells); 2 -intermediate ( $25-75 \%$ affected cells); 3 -severe ( $>75 \%$ affected cells). $0.2 \%$ DMSO (vehicle) served as a control where $0 \%$ epithelial cells were affected during culturing. $\dagger$, Insufficient number of epithelial cells present to provide reliable quantification; SAL, salinomycin; DOX, doxorubicin.

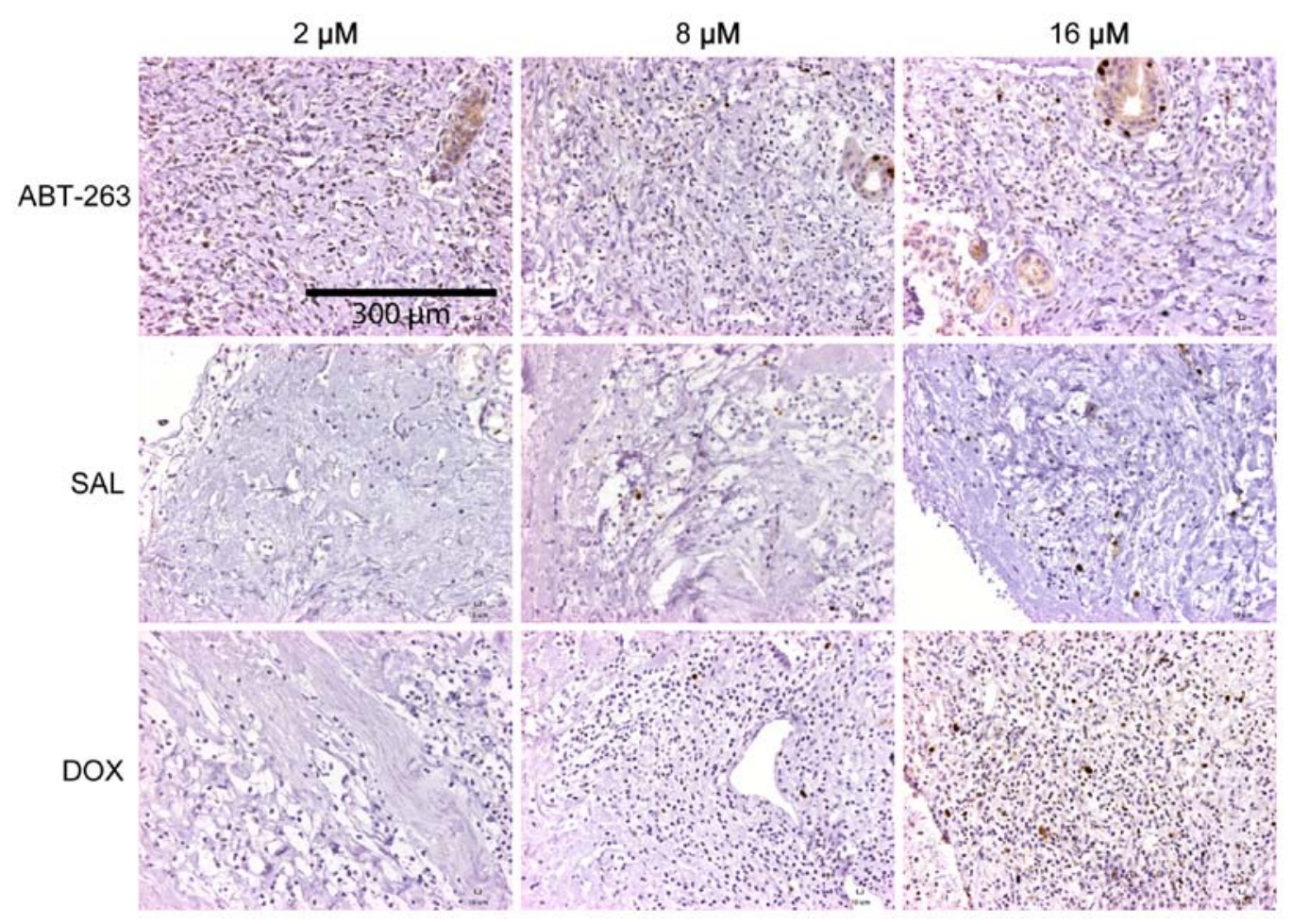

Figure 4. Anti-proliferative effects of ABT-263, SAL and DOX on breast tumors in an ex vivo model of PTB, assessed via Ki-67 immunolabeling. Images at magnification, x20.. SAL, salinomycin; DOX, doxorubicin; PTB, phyllodes tumors of the breast.

It is evident that the population of cells was mixed, with the majority showing an elongated, triangular morphology. In addition, cells with a more spherical morphology were present, as were infrequent cells with large intracellular vacuoles or vesicles. In order to confirm the presence of PT cells in this population, the expression of EpCAM (CD326), a transmembrane glycoprotein present on the surface of epithelial cells, was evaluated (35). MCF-7 and MDA-MB-231 cell lines, characterized by high and low EpCAM expression, respectively (36), were used as positive and negative controls. As shown in Fig. 7, the two breast cancer cell lines expressed EpCAM consistent with expectations, and were used to set a gate denoting high versus low expression. When the phyllodes primary cells were examined, a range of EpCAM expression was observed, with about $30 \%$ of the population in the range defined as high, indicating the presence of epithelial derived cells (Fig. 8). Once primary cells were established, they proliferated with a doubling time of 3-5 days initially, and viable cells up to passage 6 were obtained before they showed signs of slowed growth. Thus the overall number of cells obtained was limited and precluded an in-depth characterization of their properties and response to the drugs. Nonetheless, viability assays after select drug treatment were conducted using the colorimetric MTT assay (Figs. 9 and 10) (31,32). This method is based on the conversion of MTT to blue MTT-formazan crystals by mitochondrial enzymes present in viable cells. Data for DOX 


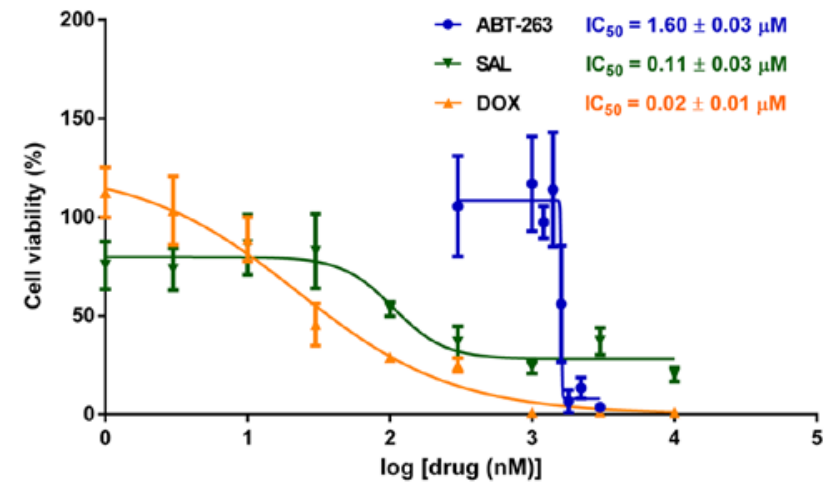

Figure 5. Cytotoxic effects of ABT-263, SAL and DOX on MCF 10A cells. MCF 10A cells were treated with vehicle (100\% viability) or increasing concentrations of ABT-263, DOX or SAL for $96 \mathrm{~h}$ and subjected to MTT viability assays. Graphed values are presented as the mean \pm standard deviation $(\mathrm{n}=4)$ and the calculated $\mathrm{IC}_{50}$ values are indicated. SAL, salinomycin; DOX, doxorubicin.

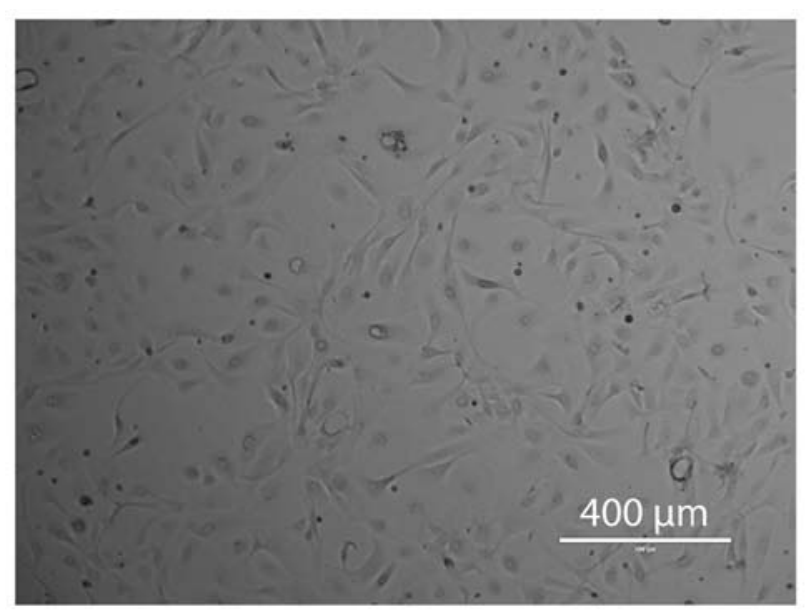

Figure 6. Phase contrast microscopy of primary phyllodes tumor cells. An image of cells at passage six is presented. The bar represents $400 \mu \mathrm{m}$ at magnification, $\mathrm{x} 10$.

using primary phyllodes cells at passage 6 are shown in Fig. 9. A concentration-dependent decrease in viability was observed with an $\mathrm{IC}_{50}$ value of $0.40 \pm 0.07 \mu \mathrm{M}$. Because of limited numbers of cells, other drugs could not be tested with a full range of concentrations, but preliminary experiments indicated that the primary PTB cells were also sensitive to ABT-263 and to SAL used at $10 \mu \mathrm{M}$, which was the highest concentration employed in MTT assay (Fig. 10).

\section{Discussion}

Surgical wide excision is the primary treatment for malignant PTBs but it frequently recurs and rarely responds to radiation or conventional chemotherapy (1-8). Although chemotherapy is not a standard PTB treatment, its application in the treatment of malignant cases would be greatly facilitated by the availability of appropriate experimental models to identify effective compounds. Primary cells derived from malignant phyllodes breast cancer have been described (37), but they are no longer available (R.K. Oldham, personal communication). In addition, although a cell line termed RW962 derived from a human phyllodes breast tumor has been reported, it was propagated as a xenograft in a mouse (38).

The present study describes two complementary laboratory models of human PTB amenable to novel drug discovery. The first involves thin tissue sections of freshly excised tumor as an experimental model to test agents active against this form of cancer. The preservation of tissue architecture and the co-existence in the same sample of both cancer and normal cells creates an extremely powerful tool for therapeutic drug screening and identification of effective as well as selective agents under uniform conditions. The activity of seven compounds was initially tested in this system including ABT-263, SAL, DOX, TAX, VCR, COL, and CIS. Three of the agents studied, namely ABT-263, SAL and DOX, were effective in killing PTB cells in the explant cultures (Figs. 2 and 3). However, the other compounds were not effective and did not result in significant induction of tumor cell death. Of possible relevance, the agents inactive in this system are cell cycle-dependent drugs. Thus TAX, VCR, and COL are all microtubule targeting agents, and the mechanism of action of CIS depends on interfering with DNA replication $(39,40)$. PTB cells are relatively slow growing and have a low proliferation index based on Ki-67 immunolabeling (Figs. 4 and S1B) (34), and this may render cell cycle-active drugs ineffective. Of the three drugs tested in the explant system that were effective, only ABT-263 was selective for the tumor cells, having only a very mild effect on normal epithelial cells even at the highest concentration of $16 \mu \mathrm{M}$ (Table I). These results are intriguing since they suggest that PTB cells may be primed for apoptosis through the intrinsic pathway (41), and indicate that further investigation of Bcl-2 inhibitors as a chemotherapeutic approach for PTB is warranted. The higher selectivity of ABT-263 for tumor versus normal cells relative to DOX and SAL was confirmed by studies with the non-tumorigenic $\mathrm{MCF}$ 10A cell line (Fig. 5).

It is of interest to compare our findings with case reports in the literature describing treatment of PTB in the clinic. Of the drugs we tested, three, namely DOX, SAL and ABT-263, were effective in killing PTB cells. Of these, only DOX has been used in the clinical setting for PTB. DOX was found to improve median survival of patients with metastatic PTB, especially when used in combination with cisplatin, cyclophosphamide, or ifosfamide $(42,43)$, and in one case complete remission was achieved with the combination of doxorubicin and cisplatin (44). Effective therapy of metastatic PTB with the combination of cisplatin and etoposide was also reported in three patients (45), measured via a reduction in tumor burden, but all three eventually died, and a larger study is needed to define response rate and median survival. These studies highlight the need for more systematic investigation of chemotherapy for PTB, and further emphasize the potential impact on drug discovery of the ex vivo and CRC models we described here. In particular, there are no reports to our knowledge testing Bcl-2 inhibition for PTB, and our finding that ABT-263 is highly effective without affecting neighboring normal cells represents an important advance. The explant system we employed represents a powerful screening tool. Importantly, several genome studies have been conducted that have provided insight into PTB pathogenesis and defined potential targets and opportunities for personalized therapeutic 

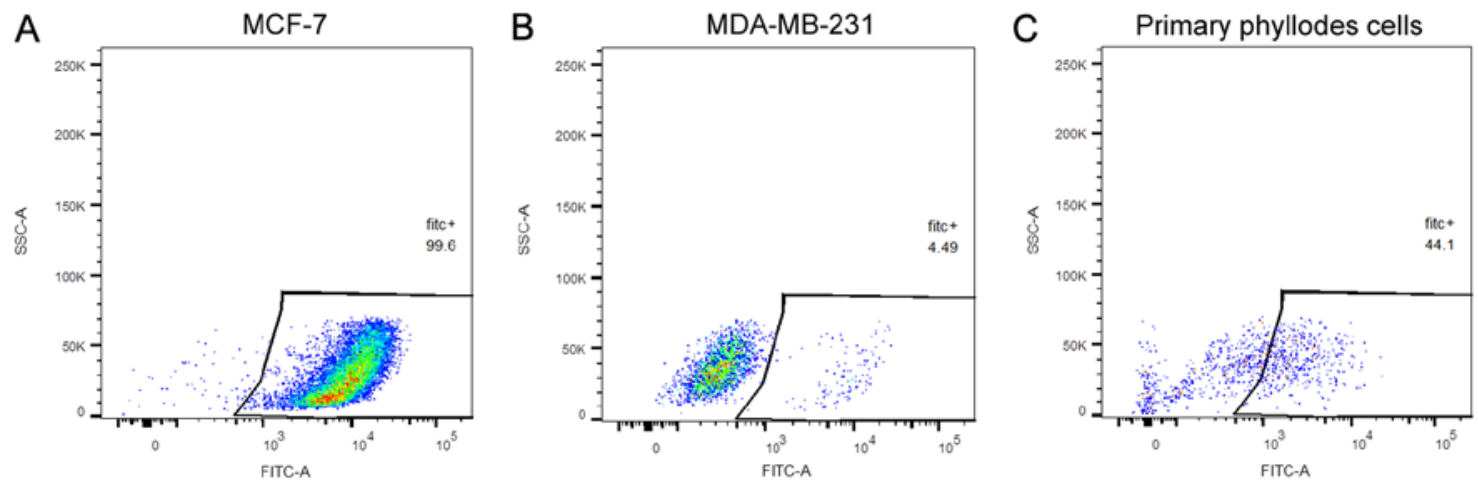

Figure 7. EpCAM expression. EpCAM expression was determined. (A) MCF-7 cell line; (B) MDA-MB-231 cell line; (C) primary PTB cells. PTB, Phyllodes tumors of the breast.

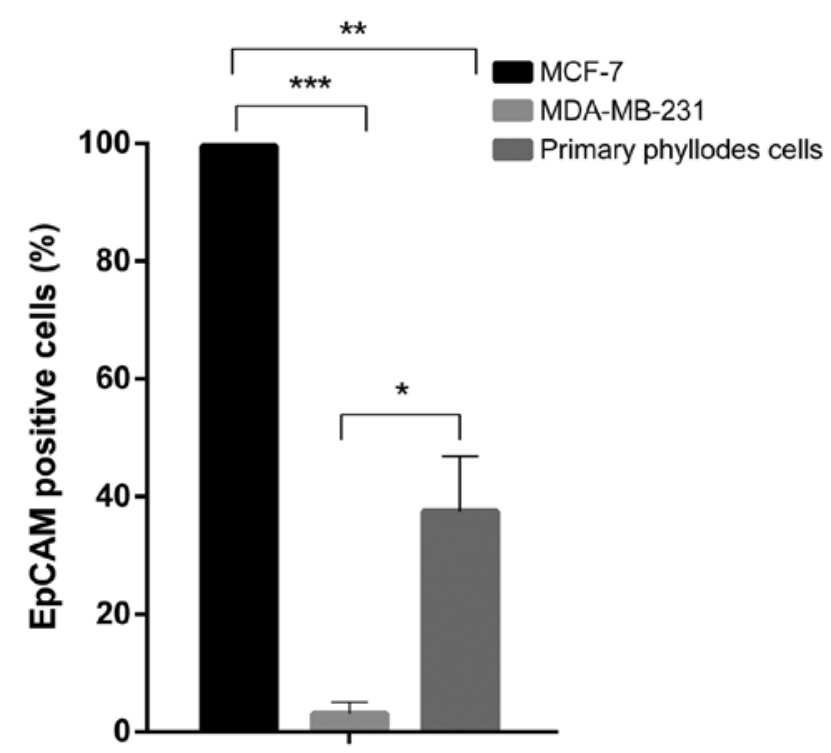

Figure 8. Summary representation of EpCAM positive cells. Quantification from two independent experiments was performed $(\mathrm{n}=2)$ and values are presented as a mean \pm standard deviation. ${ }^{*} \mathrm{P} \leq 0.05,{ }^{* *} \mathrm{P} \leq 0.01,{ }^{* * *} \mathrm{P} \leq 0.001$.

intervention (46-47). For example, the combined expression of CD34 and Bcl-2 have been shown to occur in 35-57\% of malignant PTBs analyzed (48). Data from these types of studies can suggest molecular targets for inhibition which can be readily tested in the explant system we have described here to expedite identification of drugs and drug combinations that have particular utility for treating PTBs (49).

While explants retain tissue architecture, degrees of cellularity, and specific tumor markers, the main drawback is the lack of durability, with a time-span of utility of just a few days. On the other hand, established cell lines are highly adapted to culture and there is uncertainty over how well they reflect the original tumor cells (13). Primary tumor cells represent a useful intermediate model system that offers durability without the problem of adaptation. As indicated above, while there are numerous cell lines derived from conventional forms of breast cancer, PTB cell lines or primary cell cultures are not available. In order to obtain primary PTB cells from the original tumor we followed established protocols for the generation of CRCs (21-23). Microscopic examination, EpCAM expression

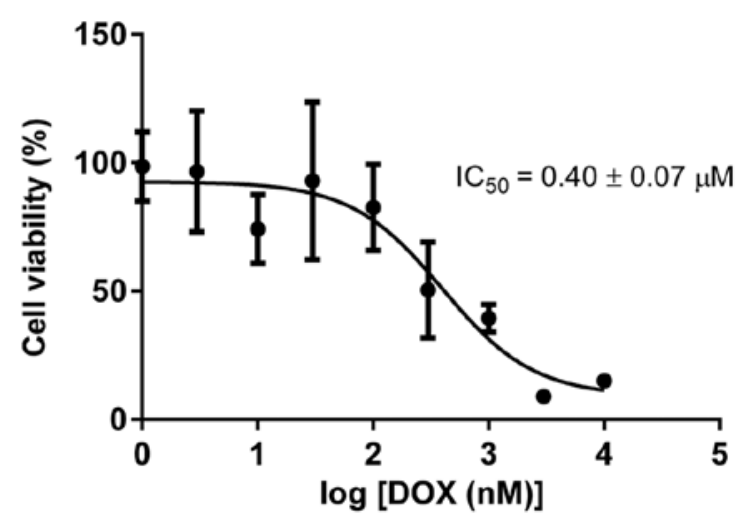

Figure 9. Sensitivity of primary phyllodes cells to DOX. Cells were treated with vehicle (100\% viability) or increasing concentrations of DOX for $96 \mathrm{~h}$ and subjected to a cell viability assay. Graphed value is presented as the mean \pm standard deviation $(n=3)$ and the calculated $\mathrm{IC}_{50}$ values are indicated. DOX, doxorubicin.

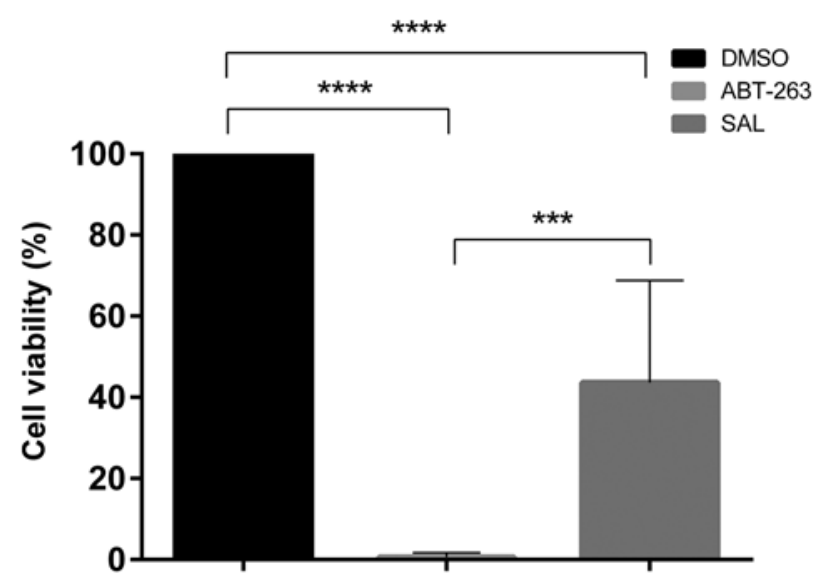

Figure 10. Sensitivity of primary phyllodes cells to ABT-263 and SAL. Cell viability was determined after treatment for $96 \mathrm{~h}$ with vehicle (0.1\% DMSO) or $10 \mu \mathrm{M}$ ABT-263 or SAL. Data represent mean \pm standard deviation $(n=3)$. ${ }^{* * * *} \mathrm{P} \leq 0.001,{ }^{* * * *} \mathrm{P} \leq 0.0001$ treatment vs. control. SAL, salinomycin.

and the proliferative nature of the population of cells obtained were consistent with the presence of PT cells. While the number of cells generated was insufficient to fully screen all of the compounds used in the explant system, the cells were 
sensitive to the three agents effective in the explant culture, namely ABT-263, DOX and SAL, strengthening the conclusion that PTB cells were present. According to published reports, CRCs typically can be passaged for extended periods (21-23), yet the primary PTB cells we generated began to show signs of slowed growth after passage 6 . Therefore further optimization of the conditions will be required to generate more robust cultures. Nonetheless, the results presented here demonstrate the feasibility of PTB drug discovery using explant and primary cultures.

\section{Acknowledgements}

The authors would like to thank Mrs. Jennifer James (UAMS Experimental Pathology Core) for processing samples for immunochemistry, Ms. Andrea Harris (UAMS Flow Cytometry Core) for performing flow cytometry experiments and assisting with interpretation and Dr Mustafa Sarimollaoglu (Department of Otolaryngology, UAMS), for assistance with the figures.

\section{Funding}

The present study was supported by funds from the Arkansas Breast Cancer Research Program.

\section{Availability of data and materials}

The datasets used and/or analyzed during the present study are available from the corresponding author on reasonable request

\section{Authors' contributions}

AU performed the ex vivo study and in vitro MTT assays, cell cultures, data preparation and analysis and wrote the draft version of manuscript, FJ acquired the primary phyllodes cells and performed the EpCAM staining experiment, $\mathrm{YY}$ analysed and quantified the H\&E stained slides and prepared the representative images. SPO analyzed the Ki-67 stained slides and prepared the representative images; AH performed the salinomycin acquisition and purification. MD performed the cell culture and performed the data analysis. TKE participated in securing funding for the project, experimental design, data interpretation and read and approved all versions of the manuscript. BMK supervised the acquisition of primary phyllodes cells and the EpCAM staining experiment, designed the experiments, interpretated results, supported manuscript writing and read and approved all versions of the manuscript. TCC supervised the project supervision, performed the data analysis, wrote the final version of the manuscript and acquired funding for the project. All authors read and approved the final manuscript.

\section{Ethics approval and consent to participate}

The protocol was determined by UAMS IRB to be 'not human subjects research' and therefore does not require IRB approval.

\section{Patient consent for publication}

Not applicable.

\section{Competing interests}

The authors declare that they have no competing interests.

\section{References}

1. Parker SJ and Harries SA: Phyllodes tumors. Postgrad Med J 77: 428-435, 2001.

2. Strode M, Khoury T, Mangieri C and Takabe K: Update on the diagnosis and management of malignant phyllodes tumors of the breast. Breast 33: 91-96, 2017.

3. Roberts N and Runk DM: Aggressive malignant phyllodes tumor. Int J Surg Case Rep 8: 161-165, 2015.

4. Mishra SP, Tiwary SK, Mishra M and Khanna AK: Phyllodes tumor of breast: A review article. ISRN Surg 2013: 361469, 2013.

5. Norat F, Dreant N, Riah Y and Lebreton E: Ann ital chir extraordinary case of malignant phylloid tumor of the breast: Surgical reconstruction treatment (Italian) 80: 475-478, 2009.

6. Chaney AW, Pollack A, McNeese MD, Zagars GK, Pisters PWT, Pollock RE and Hunt KK: Primary treatment of cystosarcoma phyllodes of the breast. Cancer 89: 1502-1511, 2000.

7. Matar N,Soumani A,Noun M,Chraibi T,Himmi A, el Mansouri A, Aderdour M and Bekkay M: Phyllodes tumors of the breast. Forty one cases. J Gynecol Obstet Biol Reprod (Paris) 26: 32-36, 1997.

8. Belkacémi Y, Bousquet G, Marsiglia H, Ray-Coquard I, Magné N, Malard Y, Lacroix M, Gutierrez C, Senkus E, Christie D, et al: Phyllodes tumor of the breast. Int J Radiat Oncol Biol Phys 70: 492-500, 2008

9. Guillot E, Couturaud B, Reyal F, Curnier A, Ravinet J, Laé M, Bollet M, Pierga JY, Salmon R and Fitoussi A: Breast cancer study group of the institut curie: Management of phyllodes breast tumors. Breast J 17: 129-137, 2011.

10. Morales-Vasquez F, Gonzalez-Angulo AM, Broglio K, Lopez-Basave HN, Gallardo D, Hortobagyi GN and De La Garza JG: Adjuvant chemotherapy with doxorubicin and dacarbazine has no effect in recurrence-free survival of malignant phyllodes tumors of the breast. Breast J 13: 551-556, 2007.

11. Hashimoto K, Mimura H, Arai Y, Doi M, Kojima Y, Tsugawa K and Nakajima Y: Successful preoperative chemoembolization in the treatment of a giant malignant phyllodes tumor. Cardiovasc Intervent Radiol 39: 1070-1075, 2016.

12. Shoemaker RH: The NCI60 human tumour cell line anticancer drug screen. Nat Rev Cancer 6: 813-823, 2006.

13. Burdall SE, Hanby AM, Lansdown MR and Speirs V: Breast cancer cell lines: Friend or foe. Breast Cancer Res 5: 89-95, 2003.

14. Witkiewicz AK, Rivadeneira DB, Ertel A, Kline J, Hyslop T, Schwartz GF, Fortina P and Knudsen ES: Association of $\mathrm{RB} /$ p16-pathway perturbations with DCIS recurrence: Dependence on tumor versus tissue microenvironment. Am J Pathol 179: 1171-1178, 2011.

15. Vargo-Gogola T and Rosen JM: Modelling breast cancer: One size does not fit all. Nat Rev Cancer 7: 659-672, 2007.

16. Dean JL, McClendon AK, Hickey TE, Butler LM, Tilley WD, Witkiewicz AK and Knudsen ES: Therapeutic response to CDK4/6 inhibition in breast cancer defined by ex vivo analyses of human tumors. Cell Cycle 11: 2756-2761, 2012.

17. Majumder B,Baraneedharan U, Thiyagarajan $S$, Radhakrishnan $P$, Narasimhan H, Dhandapani M, Brijwani N, Pinto DD, Prasath A, Shanthappa BU, et al: Predicting clinical response to anticancer drugs using an ex vivo platform that captures tumour heterogeneity. Nature Commun 6: 6169, 2015.

18. Nagourney RA: Ex vivo programmed cell death and the prediction of response to chemotherapy. Curr Treat Options Oncol 7: 103-110, 2006.

19. Kuilman T, Michaloglou C, Mooi WJ and Peeper DS: The essence of senescence. Genes Dev 24: 2463-2479, 2010.

20. Roig AI, Eskiocak U, Hight SK, Kim SB, Delgado O, Souza RF, Spechler SJ, Wright WE and Shay JW: Immortalized epithelial cells derived from human colon biopsies express stem cell markers and differentiate in vitro. Gastroenterology 138: 1012-1021, 2010.

21. Liu X, Ory V, Chapman S, Yuan H, Albanese C, Kallakury B, Timofeeva OA, Nealon C, Dakic A, Simic V, et al: ROCK inhibitor and feeder cells induce the conditional reprogramming of epithelial cells. Am J Pathol 180: 599-607, 2012.

22. Saenz FR, Ory V, AlOtaiby M, Rosenfield S, Furlong M, Cavalli LR, Johnson MD, Liu X, Schlegel R, Wellstein A and Riegel AT: Conditionally reprogrammed normal and transformed mouse mammary epithelial cells display a progenitor-cell-like phenotype. PLoS One 9: 97666, 2014. 
23. Brown DD, Dabbs DJ, Lee AV, McGuire KP, Ahrendt GM, Bhargava R, Davidson NE, Brufsky AM, Johnson RR, Oesterreich S and McAuliffe PF: Developing in vitro models of human ductal carcinoma in situ from primary tissue explants. Breast Cancer Res Treat 153: 311-321, 2015.

24. Moo TA, Sanford R, Dang C and Morrow M: Overview of breast cancer therapy. PET Clin 13: 339-354, 2018.

25. Steinmetz MO and Prota AE: Microtubule-targeting agents: Strategies to hijack the cytoskeleton. Trends Cell Biol 28 . 776-792, 2018.

26. Billard C: BH3 mimetics: Status of the field and new developments. Mol Cancer Ther 12: 1691-700, 2013.

27. Antoszczak M, Urbaniak A, Delgado M, Maj E, Borgström B, Wietrzyk J, Huczyński A, Yuan Y, Chambers TC and Strand D: Biological activity of doubly modified salinomycin analogs Evaluation in vitro and ex vivo. Eur J Med Chem 156: 510-523, 2018

28. Antoszczak M,Popiel K, Stefańska J, Wietrzyk J, Maj E, Janczak J, Michalska G, Brzezinski B and Huczynski A: Synthesis, cytotoxicity and antibacterial activity of new esters of polyether antibiotic-alinomycin. Eur J Med Chem 76: 435-444, 2014.

29. Van der Kuip H, Mürdter TE, Sonnenberg M, McClellan M, Gutzeit S, Gerteis A, Simon W, Fritz P and Aulitzky WE: Short term culture of breast cancer tissues to study the activity of the anticancer drug taxol in an intact tumor environment. BMC Cancer 6: 86, 2006.

30. Dirks WG, MacLeod RA, Nakamura Y, Kohara A, Reid Y, Milch H, Drexler HG and Mizusawa H: Cell line cross-contamination initiative: An interactive reference database of STR profiles covering common cancer cell lines. Int J Cancer 126: 303-304, 2010.

31. Alley MC, Scudiere DA, Monks A,Czerwinski M, Shoemaker RH and Boyd MR: Validation of an automated microculture tetrazolium assay (MTA) to assess growth and drug sensitivity of human tumor cell lines. Proc Am Assoc Cancer Res 27: 389-391, 1986.

32. Alley MC, Scudiere DA, Monks A, Hursey ML, Czerwinski MJ, Fine DL, Abbott BJ, Mayo JG, Shoemaker RH and Boyd MR: Feasibility of drug screening with panels of human tumor cell lines using a microculture tetrazolium assay. Cancer Res 48: 589-601, 1988

33. Yerushalmi R, Woods R, Ravdin PM, Hayes MM and Gelmon KA: Ki67 in breast cancer: Prognostic and predictive potential. Lancet Oncol 11: 174-183, 2010.

34. Lin CK, Tsai WC, Lin YC and Yu JC: Biomarkers distinguishing mammary fibroepithelial neoplasms: A tissue microarray study. Appl Immunohistochem Mol Morphol 22: 433-441, 2014.

35. Went P, Dirnhofer S, Schöpf D, Moch H and Spizzo G: Expression and prognostic significance of EpCAM. J Cancer Mol 3: 169-174, 2008.

36. Martowicz A, Spizzo G, Gastl G and Untergasser G: Phenotype-dependent effects of EpCAM expression on growth and invasion of human breast cancer cell lines. BMC Cancer 12: $501,2012$.
37. Lewko WM, Vaghmar R, Maleckar JR, Husseini S, Montgomery CA, Thurman GB and Oldham RK: Cultured breast cystosarcoma phylloides cells and applications to patient therapy. Breast Cancer Res Treat 17: 131-138, 1990.

38. Tibbetts LM, Poisson MH, Tibbetts LL and Cummings FJ: A human breast stromal sarcoma cell line with features of malignant cystosarcoma phyllodes. Cancer 62: 2176-2182, 1988.

39. Jordan MA and Wilson L: Microtubules as a target for anticancer drugs. Nat Rev Cancer 4: 253-265, 2004.

40. Wheate NJ, Walker S, Craig GE and Oun R: The status of platinum anticancer drugs in the clinic and in clinical trials. Dalton Trans 39: 8113-8127, 2010.

41. Tait SW and Green DR: Mitochondrial regulation of cell death. Cold Spring Harb Perspect Bio 5: pii a008706, 2013.

42. Mituś JW, Blecharz P, Walasek T, Reinfuss M, Jakubowicz J and Kulpa J: Treatment of patients with distant metastases from phyllodes tumor of the breast. World J Surg 40: 323-328, 2016.

43. Liu M, Yang S, Liu B, Guo L, Bao X, Liu B and Dong L: Giant malignant phyllodes tumor of the breast: A rare case report and literature review. Oncol Lett 12: 121-124, 2016.

44. Allen R, Nixon D, York M and Coleman J: Successful chemotherapy for cystosarcoma phyllodes in a young woman. Arch Intern Med 145: 1127-1128, 1985.

45. Burton GV, Hart LL, Leight GS, Iglehart JD, McCarty KS Jr and Cox EB: Cystosarcoma phyllodes effective therapy with cisplatin and etoposide chemotherapy. Cancer 63: 2088-2092, 1989.

46. Lim SZ, Ng CCY, Rajasegaran V, Guan P, Selvarajan S, Thike AA, Nasir NDBM, Koh VCY, Tan BKT, Ong KW, et al: Genomic profile of breast sarcomas: A comparison with malignant phyllodes tumours. Breast Cancer Res Treat 174: 365-373, 2019.

47. Nozad S, Sheehan CE, Gay LM, Elvin JA, Vergilio JA, Suh J, Ramkissoon S, Schrock AB, Hirshfield KM, Ali N, et al: Comprehensive genomic profiling of malignant phyllodes tumorsof the breast. Breast Cancer Res Treat 162: 597-602, 2017.

48. Moore T and Lee AH: Expression of CD34 and bcl-2 in phyllodes tumours, fibroadenomas and spindle cell lesions of the breast. Histopathology 38: 62-67, 2001.

49. Jardim DL, Conley A and Subbiah V: Comprehensive characterization of malignant phyllodes tumor by whole genomic and proteomic analysis: Biological implications for targeted therapy opportunities. Orphanet J Rare Dis 8: 112, 2013.

This work is licensed under a Creative Commons Attribution-NonCommercial-NoDerivatives 4.0 International (CC BY-NC-ND 4.0) License. 